\title{
Clinical Characteristics and Surgical Treatment of Presacral Cysts
}

\author{
Shunda Wang and Beizhan Niu* \\ Department of General Surgery, Peking Union Medical College Hospital, Chinese Academy of Medical Sciences and Peking Union Medical College, China
}

*Corresponding author: Beizhan Niu, Department of General Surgery, Peking Union Medical College Hospital, Chinese Academy of Medical Sciences and Peking Union Medical College, China.
Received Date: May 27, 2020

Published Date: June 18, 2020

\begin{abstract}
Presacral cysts are cystic lesions occurring near the sacrorectal fossa, which can be clearly diagnosed by CT and MRI and anal digital examination before surgery. The characteristics of the tumor itself and the special location are not conducive to surgical resection. The introduction of laparoscopic techniques has brought hope for treatment, but attention should be paid to avoid damage to the rectal and presacral venous plexus. Presacral cysts with insidious onset should be checked regularly and discovered timely. Patients have good prognosis. Multi-disciplinary diagnosis was necessary. This review aims to summarize the clinical characteristics, diagnostic methods, surgical treatment, and prognosis of presacral cysts.
\end{abstract}

Keywords: Presacral cyst; Surgery; Diagnosis; Treatment

\section{Introduction}

Presacral cysts are cystic masses that occur in the sacral and rectal spaces [1]. The space is anterior to the rectum, anterolateral to the rectal ligament, bilateral to the iliac vessels and ureters, posterior to the sacrum and coccyx, superior to the rectum, bladder, or uterine depression, and inferior to the coccygeal ligament, levator ani muscle, and coccygeal muscle. The space contains loose connective tissue, branches of sacral plexus, high branches of sympathetic nerve, blood vessels and other tissues [2]. Clinically, presacral cysts in adult are uncommon, usually deep in location and not easy to be detected early. The clinical symptoms of presacral cysts are related to the location, size, concurrent infection, and the degree of compression or involvement of adjacent organs. 50\% of patients with presacral cysts are asymptomatic [3].

\section{Clinical Symptoms}

The onset of presacral cyst is concealed and develops slowly, without any symptoms in the early stage. When the cystic mass develops to a certain extent, its symptoms are mostly related to compression of adjacent organs and tissues. Because the cyst is mostly a benign mass, the pain caused by it is not severe, mostly pain with abdominal distension. The most common symptoms are lower abdominal pain, low back pain, lower abdominal mass, defecation, dysuria, palpable tumors in buttocks and abnormal sensation in perineum. Additionally, some cases are found presacral tumors by physical examination without obvious symptoms, especially by gynecological and obstetric physical examination.

\section{Diagnostic Methods}

All patients with presacral cysts should receive digital rectal examination. Tumors were usually palpable on the posterior wall of the rectum, which was soft, partly hard, movable, and no blood staining found in the finger retraction cuff. Digital rectal examination is the simplest and convenient noninvasive examination for presacral masses. It was reported that the detection rate of digital rectal examination for lesions could reach $67-96 \%$ [4-6]. Because of the special location of presacral cyst, 
puncture cytology is not advocated to avoid secondary infection of cyst and injury of intestine, bladder, and blood vessel. Moreover, pelvic B-ultrasound examination is a cheap, non-invasive, and high positive rate examination method for presacral cysts. B-ultrasound examination can preliminarily determine the size, location, and nature of the lesions. the pelvic B-ultrasound examination could detect the hypoechoic mass in the presacral area, suspicious cystic space occupying, irregular shape with clear boundary (Figure 1).
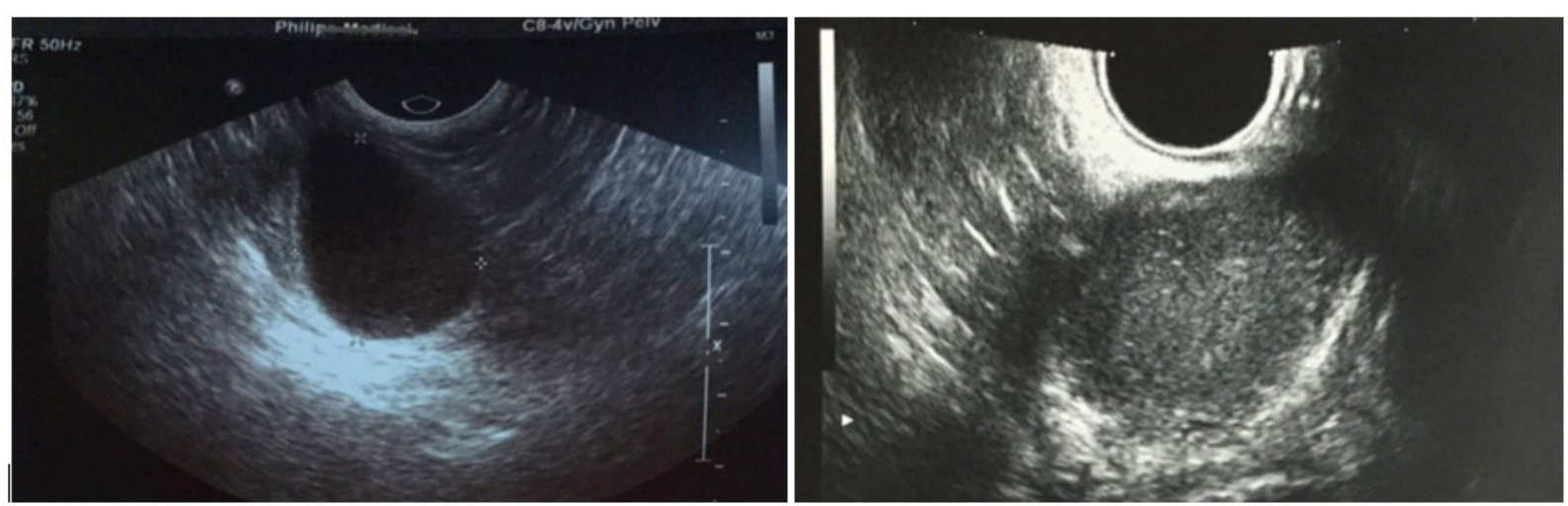

Figure 1: Typical image of presacral mass under B-mode ultrasound examination.

However, B-ultrasound is not clear for the adhesion between cysts and surrounding tissues, while pelvic CT examination can determine whether the tumor adheres to the rectal wall, or whether it invades blood vessels. In addition, enhanced CT can also help distinguish benign from malignant tumors [7]. Enhanced CT examination could reveal a round cystic lesion in the soft tissue of the anterior sarco- coccyx with uneven density. The CT value was normally 9-40 HU and the capsule might intact without calcification. Hemorrhage and calcification could be seen in some larger cysts, which could be slightly enhanced. Pelvic MRI is beneficial for presacral soft tissue imaging and has no radiation damage compared with CT. As for MRI examination, cystic solid signals with long $\mathrm{T} 1$ and $\mathrm{T} 2$ can be seen in the presacral region, with clear boundary, intact capsule without enhancement.

\section{Operation and Treatment}

Surgery is an effective treatment for presacral cysts. There are three traditional surgical methods for presacral cysts: transsacrococcygeal, trans-abdominal and trans-sacroabdominal $[8,9]$. Studies have shown that trans sacrococcygeal approach can obtain better prognosis for tumors with small diameter and palpable border on digital rectal examination. With the trans-sacrococcygeal approach, the surgical field is wider, the risk of injury to the intestinal wall is small, and the patient recovers faster [10]. At present, there are two main surgical methods, namely laparoscopic surgery, and open surgery. (1) Laparoscopic surgery: the patient in lithotomy position and $2 \mathrm{ml}$ methylene blue was injected through the anus. After the establishment of pneumoperitoneum, laparoscopic exploration was performed first, and the peritoneum around the rectum was lifted. After the cyst was found, the space between the cyst and the surrounding normal tissues was cut along the cyst wall to avoid damaging the rectum and vein. After separation, the cyst was completely cut off. (2) Open surgery: A trans sacrococcygeal approach was adopted. The skin of sacrococcygeal region was cut longitudinally, and the incision was about $1 \mathrm{~cm}$ from the side of coccyx to above the anal margin. The cyst was exposed to the maximum extent. During the operation, attention was paid to avoid damaging the rectum. If the cyst wall was clearly demarcated, the cyst wall should be separated and resected closely. In case of severe adhesion around cyst wall, residual cyst wall tissue should also be prevented.

Due to conventional open surgery with high trauma. laparoscopic surgery has replaced traditional surgery in recent years [11]. Laparoscopic resection of presacral cyst could shorten the operation time, lessen intraoperative bleeding, and reduced the postoperative complications compared to the conventional open surgery. There are few cases of sacral cyst treated by laparoscopy reported in literatures, but the clear visual field and minimally invasive of laparoscopy cannot be replaced.

\section{Complications}

The main complications of the operation for presacral cyst are massive hemorrhage of wound surface and side injury. Wound hemorrhage is mainly divided into two types: massive exudation of blood from the wound and bleeding from iliac vascular injury. Massive bleeding on the face is often caused by rupture of presacral vein, bleeding, or blunt separation of the wound. Therefore, we advocate that presacral cysts should be separated as sharply as possible and forceps should be avoided on the sacral surface. Iliac vascular injury and bleeding, mostly due to cysts and iliac vessels adhesions, especially thin walls of iliac veins, blunt detachment, or sharpness separation can cause venous wall rupture and bleeding. The incidence of intraoperative side injuries is lower than that of intraoperative hemorrhage, mainly rectal injuries, therefore the 
built-in thick anal canal is conducive to the identification of the rectum during operation and can reduce its damage. Moreover, difficulty in defecation or urination after operation, even sensory disturbance in perineal area after operation, may be caused by injury of ureter and nerve plexus and other tissues during blunt separation during operation.

\section{Pathology}

As for the pathological pattern of presacral cysts, epidermoid cyst, mature teratoma and dermoid cyst were relatively common
(Figure 2). Especially, presacral cysts are mostly epidermoid cysts with no obvious symptoms in the early stage, and their clinical manifestations are affected by the size and nature of the tumors and the degree of compression or invasion of surrounding organs. Epidermoid cysts may occur as a result of implantation of tiny debris into the skin during trauma or as ectopic remnants from closure of the neural groove in the early embryonic stage [12]. Presacral teratoma is a low-grade malignancy with low incidence. Early detection and timely treatment can obtain a good prognosis.

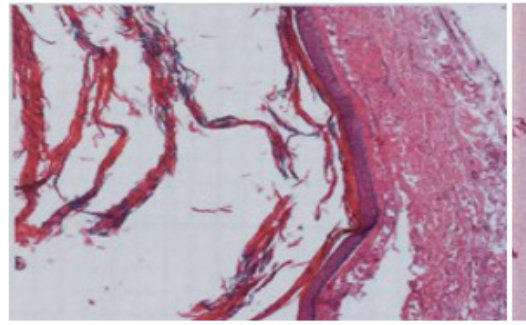

A

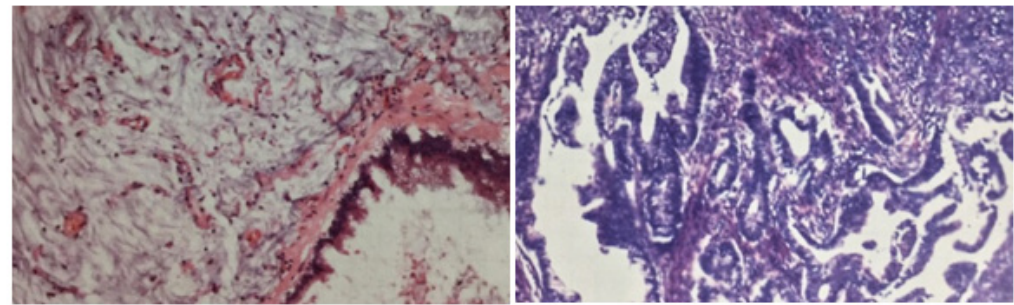

C

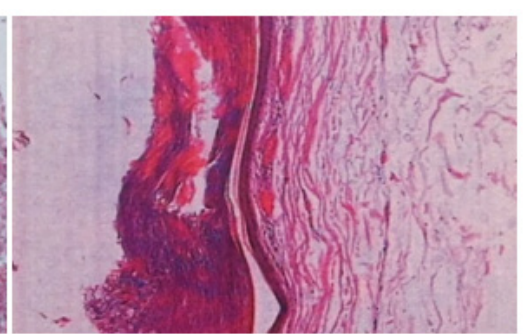

B

D

Figure 2: Microscopic pathological section of epidermoid cyst (A, B) and teratoma (C, D).

\section{Conclusion}

Presacral cysts refer to posterior rectal masses in adults and are more common in middle-aged women, with a male-to-female ratio of 1:3 [13]. The onset of presacral cyst is concealed, mostly without obvious symptoms, or accompanied by lower abdominal mass, lower abdominal pain, diarrhea, low back pain, urinary symptoms, and perineal dysesthesia, etc. Regular physical examination helps to screen out the lesions in the presacral area. Some patients were found presacral mass during routine gynecological examination. It is suggested that gynecologists and obstetricians should also pay enough attention to presacral mass besides paying attention to uterine double adnexal lesions. B-ultrasonography should be routinely performed in patients with presacral cysts, and CT and MRI can be used as auxiliary examinations. Laparoscopic resection of presacral lesions is recommended, and attention should be paid to the protection of blood vessels, nerves, and rectum in the presacral area. Postoperative prognosis was good, and most of the pathological types were epidermoid cysts. In addition, presacral cysts often occur in female and some patients are found during routine gynecological examinations. Therefore, it is suggested that multidisciplinary treatment of presacral cysts should be adopted to achieve early detection and treatment.

\section{Acknowledgement}

None.

\section{Conflict of Interest}

No conflict of interest.

\section{References}

1. Hobson KG, Ghaemmaghami V, Roe JP, Goodnight JE, Khatri VP (2005) Tumors of the retrorectal space. Dis Colon Rectum 48(10): 1964-1974.

2. Jang SH, Jang KS, Song YS, Kyueng-Whan Min, Hong-Xiu Han, et al. (2006) Unusual prerectal location of a tailgut cyst: a case report. World J Gastroenterol 12(31): 5081-5083.

3. Kanemitsu T, Kojima T, Yamamoto S, Koike A, Takeshige K, et al. (1993) The trans-sphincteric and trans-sacral approaches for the surgical excision of rectal and presacral lesions. Surg Today 23(10): 860-866.

4. Glasgow SC, Birnbaum EH, Lowney JK, James W Fleshman, Ira J Kodner, et al. (2005) Retrorectal tumors: a diagnostic and therapeutic challenge. Dis Colon Rectum 48(8): 1581-1587.

5. Feldenzer JA, McGauley JL, McGillicuddy JE (1989) Sacral and presacral tumors: problems in diagnosis and management. Neurosurgery 25(6): 884-891. 
6. Testini M, Catalano GJ, Macarini L, Paccione F (1996) Diagnosis and surgical treatment of retroperitoneal tumours. Int Surg 81(1): 88-93.

7. Dahan H, Arrive L, Wendum D, Docou LPH, Djouhri H, et al. (2001) Retrorectal developmental cysts in adults: clinical and radiologichistopathologic review, differential diagnosis, and treatment. Radiographics 21(3): 575-584

8. Pidala MJ, Eisenstat TE, Rubin RJ, Salvati EP (1999) Presacral cysts: transrectal excision in select patients. Am Surg 65(2): 112-115.

9. Singer MA, Cintron JR, Martz JE, Schoetz DJ, Abcarian H (2003) Retrorectal cyst: a rare tumor frequently misdiagnosed. J Am Coll Surg 196(6): 880-886

10. Abel ME, Nelson R, Prasad ML, Pearl RK, Orsay CP, et al. (1985) Parasacrococcygeal approach for the resection of retrorectal developmental cysts. Dis Colon Rectum 28(11): 855-858.
11. Lim SW, Huh JW, Kim YJ, Kim HR (2011) Laparoscopy-assisted resection of tailgut cysts: report of a case. Case Rep Gastroenterol 5(1): 22-27.

12. Lev-Chelouche D, Gutman M, Goldman G, Einat Even-Sapir, Isaac Meller et al. (2003) Presacral tumors: a practical classification and treatment of a unique and heterogeneous group of diseases. Surgery 133(5): 473 478.

13. Dahan H, Arrive L, Wendum D, Docou LPH, Djouhri H, et al. (2001) Retrorectal developmental cysts in adults: clinical and radiologichistopathologic review, differential diagnosis, and treatment. Radiographics 21(3): 575-584. 